\title{
AURC Infinity Predicted Normalized by Weight
}

National Cancer Institute

\section{Source}

National Cancer Institute. AURC Infinity Predicted Normalized by Weight. NCI Thesaurus. Code C92361.

The area under the urinary excretion rate curve (AURC) extrapolated to infinity, based on the last predicted excretion rate, divided by the weight. 